\title{
Long time behavior of solutions to semilinear parabolic equations involving strongly degenerate elliptic differential operators
}

\author{
P. T. Thuy and N. M. Tri
}

\begin{abstract}
In this paper we consider initial boundary value problem for semilinear parabolic equations involving strongly degenerate elliptic differential operators. Depending on the concrete types of nonlinearity we establish the existence of compact connected global attractors of semigroups generated by the problem under consideration.
\end{abstract}

Mathematics Subject Classification (2010). Primary 35B41; Secondary 35K65, 35D05.

Keywords. Semilinear parabolic equations, Strongly degenerate elliptic differential operators, Long-time behavior of solutions, Global solutions, Global attractors.

\section{Introduction}

A large number of authors have studied the global existence and the longtime behavior of solutions to semilinear parabolic equations (see, for example $[1-3]$, and the references therein). In recent years, many works have been devoted to the study of the long-time behavior of solutions to semilinear degenerate parabolic equations (see $[4,5]$ and the references therein). In the recent papers $[6,7]$, the authors of this article have studied a boundary value problem for the following strongly degenerate elliptic differential operator

$$
P_{\alpha, \beta} u:=\Delta_{x} u+\Delta_{y} u+|x|^{2 \alpha}|y|^{2 \beta} \Delta_{z} u
$$

where $(x, y, z)=\left(x_{1}, \ldots, x_{N_{1}}, y_{1}, \ldots, y_{N_{2}}, z_{1}, \ldots, z_{N_{3}}\right) \in \Omega \subset \mathbb{R}^{N_{1}+N_{2}+N_{3}}$, $\alpha, \beta \geq 0, \alpha+\beta>0$, 


$$
\begin{aligned}
\Delta_{x} & =\sum_{i=1}^{N_{1}} \frac{\partial^{2}}{\partial x_{i}^{2}}, \Delta_{y}=\sum_{j=1}^{N_{2}} \frac{\partial^{2}}{\partial y_{j}^{2}}, \Delta_{z}=\sum_{l=1}^{N_{3}} \frac{\partial^{2}}{\partial z_{l}^{2}}, \\
|x|^{2 \alpha} & =\left(\sum_{i=1}^{N_{1}} x_{i}^{2}\right)^{\alpha},|y|^{2 \beta}=\left(\sum_{j=1}^{N_{2}} y_{j}^{2}\right)^{\beta}
\end{aligned}
$$

and $\Omega$ is a bounded domain with smooth boundary in $\mathbb{R}^{N_{1}+N_{2}+N_{3}}$. Note that the operator $P_{\alpha, \beta}$ under consideration is strongly degenerate elliptic, i. e. degenerate on the union of two intersecting surfaces. We associate the operator $P_{\alpha, \beta}$ with the weighted Sobolev space $S_{1}^{p}(\Omega), 1 \leq p<\infty$, consisting of all functions $u \in L^{p}(\Omega)$ such that $\nabla_{x} u, \nabla_{y} u,|x|^{\alpha}|y|^{\beta} \nabla_{z} u \in L^{p}(\Omega)$. For the norm in $S_{1}^{p}(\Omega)$ we take

$\|u\|_{S_{1}^{p}(\Omega)}^{p}=\int_{\Omega}\left(|u|^{p}+\sum_{i=1}^{N_{1}}\left|\frac{\partial u}{\partial x_{i}}\right|^{p}+\sum_{j=1}^{N_{2}}\left|\frac{\partial u}{\partial y_{j}}\right|^{p}+\left.\left.\sum_{l=1}^{N_{3}}|| x\right|^{\alpha}|y|^{\beta} \frac{\partial u}{\partial z_{l}}\right|^{p}\right) \mathrm{d} x \mathrm{~d} y \mathrm{~d} z$,

where

$$
\mathrm{d} x=\mathrm{d} x_{1} \ldots \mathrm{d} x_{N_{1}}, \quad \mathrm{~d} y=\mathrm{d} y_{1} \ldots \mathrm{d} y_{N_{2}}, \quad \mathrm{~d} z=\mathrm{d} z_{1} \ldots \mathrm{d} z_{N_{3}} .
$$

If $\mathrm{p}=2$ we can also define the scalar product in $S_{1}^{2}(\Omega)$ as follows

$$
\begin{aligned}
(u, v)_{S_{1}^{2}(\Omega)}= & (u, v)_{L^{2}(\Omega)}+\left(\nabla_{x} u, \nabla_{x} v\right)_{L^{2}(\Omega)}+\left(\nabla_{y} u, \nabla_{y} v\right)_{L^{2}(\Omega)} \\
& +\left(|x|^{\alpha}|y|^{\beta} \nabla_{z} u,|x|^{\alpha}|y|^{\beta} \nabla_{z} v\right)_{L^{2}(\Omega)} .
\end{aligned}
$$

The space $S_{1,0}^{p}(\Omega)$ is defined as the closure of $C_{0}^{1}(\Omega)$ in the space $S_{1}^{p}(\Omega)$, where $C_{0}^{1}(\Omega)$ denotes the set of functions in $C^{1}(\Omega)$ with a compact support in $\Omega$. Imbedding theorems for the Sobolev space associate with the operator $P_{\alpha, \beta}$ were established in [7] (see also [8] for imbedding theorems for weighted Sobolev spaces associated with nonsmooth vector fields). The review of recent investigations on local and global properties of solutions to semilinear degenerate elliptic equations is available in $[9,10]$. In the present paper we deal with the initial boundary value problem

$$
\begin{gathered}
\frac{\partial u}{\partial t}-P_{\alpha, \beta} u+f(x, y, z, u)=0,(x, y, z) \in \Omega, t>0, \\
u(x, y, z, t)=0,(x, y, z) \in \partial \Omega, t>0, \\
u(x, y, z, 0)=u_{0}(x, y, z),(x, y, z) \in \Omega,
\end{gathered}
$$

where $f \in C(\Omega \times \mathbb{R} ; \mathbb{R})$. The results obtained here generalize the results in $[4,5]$ in two aspects: in the more complicated character of degeneracy of the linear operator and more complicated structure of the nonlinear term. The solutions (mild or weak) of the problem (1)-(3) are described with the help of continuous semigroups $S(t)$ which act on $S_{0}^{1}(\Omega)$ or on $L^{2}(\Omega)$. We will consider separately the cases when $S(t)$ is a gradient system and when it is not a gradient system. The rest of the paper is organized as follows. In the second part we deal with the case when 
the nonlinear term has under-critical growth at infinity. In the third part we study the case when the nonlinear term has big growth at infinity. Throughout the paper we denote by $C$ a general constant which may vary from place to place and it can take different values even in one line. Put

$$
N_{\alpha, \beta}=N_{1}+N_{2}+(\alpha+\beta+1) N_{3}, F(x, y, z, u)=\int_{0}^{u} f(x, y, z, \tau) \mathrm{d} \tau .
$$

\section{Gradient systems}

Let us first briefly introduce some notations and preliminary results. Denote by $(.,$.$) the scalar product in L^{2}(\Omega)$. The operator $A:=-P_{\alpha, \beta}$ is positive, self-adjoint with domain of definition defined by

$$
D(A)=\left\{u \in S_{0}^{1}(\Omega): A(u) \in L^{2}(\Omega)\right\} .
$$

It can be shown that there exists a complete in $L^{2}(\Omega)$ system of eigenvectors of $A$ :

$$
\begin{aligned}
A\left(e_{j}\right) & =\lambda_{j} e_{j}, \quad\left(e_{j}, e_{k}\right)_{L^{2}(\Omega)}=\delta_{j, k} ; \quad j, k=1,2, \ldots \\
0 & <\lambda_{1} \leq \lambda_{2} \leq \cdots \leq \lambda_{j} \leq \ldots ; \lim _{j \rightarrow \infty} \lambda_{j}=+\infty
\end{aligned}
$$

where $\delta_{j, k}$ is the Kronecker symbol. For any $\theta \in \mathbb{R}$ let $D^{\theta}$ be the domain of definition of $A^{\theta}$. We can define the scalar product on $D^{\theta}$

$$
(u, v)_{D^{\theta}}=\left(A^{\theta} u, A^{\theta} v\right)_{L^{2}(\Omega)}
$$

which will turn $D^{\theta}$ into a Hilbert space. One can easily verify that $D^{\theta_{1}}$ is imbedded into $D^{\theta_{2}}$ if $\theta_{1} \geq \theta_{2}$; moreover the imbedding is compact if $\theta_{1}>\theta_{2}$. With the above notations it is readily seen that

$$
\begin{array}{r}
D^{\frac{1}{2}}=S_{0}^{1}(\Omega), D^{0}=L^{2}(\Omega) ; \\
A^{\eta}: D^{\theta} \longrightarrow D^{\theta-\eta}, A^{\theta_{1}+\theta_{2}}=A^{\theta_{1}} A^{\theta_{2}} .
\end{array}
$$

In this section we will impose the following conditions on the nonlinear term $f(x, y, z, u)$ : there are positive constants $\rho, \mu, C$, a function $g(x, y, z) \in L^{1}(\Omega)$ such that $\mu<\lambda_{1}$ and

$$
\begin{aligned}
& |f(x, y, z, u)-f(x, y, z, v)| \leq C|u-v|\left(1+|u|^{\rho}+|v|^{\rho}\right) \\
& \text { with } \quad \frac{2}{N_{\alpha, \beta}-2}<\rho<\frac{4}{N_{\alpha, \beta}-2}, \\
& f(x, y, z, 0) \in L^{p}(\Omega) \text { for every } p \in\left[1, \frac{2 N_{\alpha, \beta}}{\left(N_{\alpha, \beta}-2\right)(1+\rho)}\right), \\
& F(x, y, z, u) \geq-\frac{\mu u^{2}}{2}-g(x, y, z), \\
& f(x, y, z, u) u \geq-\mu u^{2}-g(x, y, z) .
\end{aligned}
$$

We will show that the problem (1)-(3) then generates a semigroup which posseses a strict Lyapunov functional on $S_{0}^{1}(\Omega)$, which means that the semigroup $S(t)$ is a continuous gradient system on $S_{0}^{1}(\Omega)$. Put $2_{\alpha, \beta}^{*}=\frac{2 N_{\alpha, \beta}}{N_{\alpha, \beta}-2}$. The 
conditions (4), (5) on $\rho$ and $p$ is then rephrased as $\frac{2_{\alpha, \beta}^{*}-2}{2}<\rho<2_{\alpha, \beta}^{*}-2$, $p \in\left[1, \frac{2_{\alpha, \beta}^{*}}{1+\rho}\right)$. With the help of the interpolation property of fractional powers and the Holder inequality it is not difficult to show

Lemma 1. For every $p \in\left(\frac{2_{\alpha, \beta}^{*}}{2_{\alpha, \beta}^{*}-1}, 2\right]$, the space $L^{p}(\Omega)$ is continuously imbedded into $D^{-\gamma}$ with $\left(\gamma>\frac{2_{\alpha, \beta}^{*}(2-p)}{2 p\left(2_{\alpha, \beta}^{*}-2\right)}\right)$.

Proof. From [7] we have the following continuous imbedding maps for every $\varepsilon \in\left(0,2_{\alpha, \beta}^{*}-2\right]$

$$
\begin{aligned}
D^{\frac{1}{2}}=S_{0}^{1}(\Omega) & \subset L^{2_{\alpha, \beta}^{*}-\varepsilon}(\Omega), \\
& L^{2}(\Omega) \subset L^{2}(\Omega) .
\end{aligned}
$$

Hence, by an interpolation theorem (see for example [11]) we deduce that $D^{\frac{\left(2_{\alpha, \beta}^{*}-\varepsilon\right)(2-p)}{2\left(2_{\alpha, \beta}^{*}-2-\varepsilon\right)}}(\Omega)$ is imbedded into $L^{\frac{p}{p-1}}(\Omega)$ for $p \in\left(\frac{2_{\alpha, \beta}^{*}}{2_{\alpha, \beta}^{*}-1}, 2\right]$ and every $\varepsilon \in$ $\left(0,2_{\alpha, \beta}^{*}-2\right]$. Now the conclusion of the lemma follows from the duality argument.

Remark 1. Note that in the above lemma $0 \leq \frac{2_{\alpha, \beta}^{*}(2-p)}{2 p\left(2_{\alpha, \beta}^{*}-2\right)}<\frac{1}{2}$ if $p \in\left(\frac{2_{\alpha, \beta}^{*}}{2_{\alpha, \beta}^{*}-1}, 2\right]$.

Lemma 2. Suppose that $f(x, y, z, u)$ satisfies the conditions (4), (5). Then the Nemytskii map

$$
\begin{aligned}
& f: D^{\frac{1}{2}} \longrightarrow D^{-\gamma_{0}} \\
& u \longrightarrow f(x, y, z, u(x, y, z)),
\end{aligned}
$$

where $\gamma_{0}=\frac{\rho}{2\left(2_{\alpha, \beta}^{*}-2\right)}$, is Lipschitzian on every bounded set of $D^{\frac{1}{2}}$.

Proof. Choose

$$
p_{0}=\frac{2_{\alpha, \beta}^{*}\left(2_{\alpha, \beta}^{*}+3 \rho+2\right)}{2(1+\rho)\left(2_{\alpha, \beta}^{*}+\rho\right)} .
$$

In view of (2) it is easily seen that

$$
\frac{2_{\alpha, \beta}^{*}}{2_{\alpha, \beta}^{*}-1}<p_{0}<\frac{2_{\alpha, \beta}^{*}}{1+\rho}<2, \quad \frac{2_{\alpha, \beta}^{*}\left(2-p_{0}\right)}{2 p_{0}\left(2_{\alpha, \beta}^{*}-2\right)}<\frac{\rho}{2\left(2_{\alpha, \beta}^{*}-2\right)} .
$$

By Lemma $1, L^{p_{0}}(\Omega)$ is continuously imbedded into $D^{-\gamma_{0}}$. Therefore it suffices to prove that $f: D^{\frac{1}{2}} \longrightarrow L^{p_{0}}(\Omega)$ is a Lipschitzian map on every bounded set of $D^{\frac{1}{2}}$. First, we prove that $f(x, y, z, u(x, y, z)) \in L^{p_{0}}(\Omega)$ if $u(x, y, z) \in D^{\frac{1}{2}}$. Indeed, by putting $v=0$ into (4) we have

$$
|f(x, y, z, u)| \leq C\left(1+|u|^{1+\rho}\right)+|f(x, y, z, 0)|,
$$

from which it implies that 


$$
|f(x, y, z, u)|^{p_{0}} \leq C\left(1+|u|^{(1+\rho) p_{0}}+|f(x, y, z, 0)|^{p_{0}}\right) .
$$

Therefore by an imbedding theorem of [7],

$$
\left(\int_{\Omega}|f(x, y, z, u)|^{p_{0}} \mathrm{~d} x \mathrm{~d} y \mathrm{~d} z\right)^{\frac{1}{p_{0}}} \leq C\left(1+\|u\|_{D^{\frac{1}{2}}}+\|f(x, y, z, 0)\|_{L^{p_{0}}(\Omega)}\right)<\infty .
$$

Next, we prove that the Nemytskii map $f$ is a Lipschitzian on every bounded set of $D^{\frac{1}{2}}$. Fix a number $r$ and take two arbitrary elements $u, v \in D^{\frac{1}{2}}$ such that $\max \left\{\|u\|_{D^{\frac{1}{2}}},\|v\|_{D^{\frac{1}{2}}}\right\}<r$. We have

$$
\begin{aligned}
& \int_{\Omega}|f(x, y, z, u)-f(x, y, z, v)|^{p_{0}} \mathrm{~d} x \mathrm{~d} y \mathrm{~d} z \\
& \quad \leq C \int_{\Omega}\left(|u-v|^{p_{0}}+|u|^{p_{0} \rho}|u-v|^{p_{0}}+|v|^{p_{0} \rho}|u-v|^{p_{0}}\right) \mathrm{d} x \mathrm{~d} y \mathrm{~d} z .
\end{aligned}
$$

By the Holder inequality

$$
\begin{aligned}
& \int_{\Omega}|u|^{p_{0} \rho}|u-v|^{p_{0}} \mathrm{~d} x \mathrm{~d} y \mathrm{~d} z \leq\left\||u|^{p_{0} \rho}\right\|_{L^{\frac{1+\rho}{\rho}(\Omega)}}\left\||u-v|^{p_{0}}\right\|_{L^{1+\rho}(\Omega)} \\
& \quad:=\|u\|_{L^{p_{0}(1+\rho)}(\Omega)}^{p_{0} \rho}\|u-v\|_{L^{p_{0}(1+\rho)}(\Omega)}^{p_{0}} \leq r^{p_{0} \rho}\|u-v\|_{L^{p_{0}(1+\rho)}(\Omega)}^{p_{0}} .
\end{aligned}
$$

Analogously

$$
\int_{\Omega}|v|^{p_{0} \rho}|u-v|^{p_{0}} \mathrm{~d} x \mathrm{~d} y \mathrm{~d} z \leq r^{p_{0} \rho}\|u-v\|_{L^{p_{0}(1+\rho)}(\Omega)}^{p_{0}} .
$$

Hence

$\|f(x, y, z, u)-f(x, y, z, v)\|_{L^{p_{0}}(\Omega)} \leq C_{1}(r)\|u-v\|_{L^{p_{0}(1+\rho)}(\Omega)} \leq C_{2}(r)\|u-v\|_{D^{\frac{1}{2}}} \cdot$

With help of Lemma 2 and the same method as in [12] it is not difficult to establish the following

Proposition 1. Assume that $f$ satisfies the conditions (4)-(7). Then for any $R>0$ there exists $T:=T(R)>0$ sufficiently small such that for $u_{0} \in$ $D^{\frac{1}{2}},\left\|u_{0}\right\|_{D^{\frac{1}{2}}} \leq R$ the problem $(1)-(3)$ has a unique solution $u \in C\left([0, T] ; D^{\frac{1}{2}}\right)$. Moreover $u_{t}(t) \in D^{\delta}$ for each $t \in(0, T)$ and $\delta<\frac{\rho+2_{\alpha, \beta}^{*}-2}{2\left(2_{\alpha, \beta}^{*}-2\right)}$.

From (4) it implies that

$$
|F(x, y, z, u)| \leq C\left(1+|u|^{2+\rho}+|f(x, y, z, 0) \| u|\right) .
$$

Hence

$$
\int_{\Omega}|F(x, y, z, u)| \mathrm{d} x \mathrm{~d} y \mathrm{~d} z \leq C\left(1+\|u\|_{L^{2+\rho}(\Omega)}\right) .
$$

Therefore it makes sense to introduce the Lyapunov functional defined on the trajectory of the solution found in Proposition 1

$$
\Phi(u)=\frac{1}{2}\|u\|_{D^{\frac{1}{2}}}^{2}+\int_{\Omega} F(x, y, z, u) \mathrm{d} x \mathrm{~d} y \mathrm{~d} z .
$$


By multiplying (1) with $\frac{\partial u}{\partial t}$ and integrating over $\Omega$ we have

$$
\frac{\mathrm{d}}{\mathrm{d} t} \Phi(u(t))=-\left\|u_{t}(t)\right\|_{L^{2}(\Omega)}^{2} \leq 0 .
$$

Proposition 2. Assume that $f$ satisfies the conditions (4)-(7) and $u_{0} \in D^{\frac{1}{2}}$. Then the problem (1)-(3) has a unique global solution $u \in C\left([0, \infty) ; D^{\frac{1}{2}}\right)$.

Proof. The uniqueness of the solution was stated in Proposition 1. We will show that the local solution in Proposition 1 can be extended globally in time. From (8) it follows that the solution $u(t)$ on the interval of maximal existence $\left[0, T_{\max }\right)$ satisfies

$\Phi(u(0)) \geq \Phi(u(t)) \geq \frac{1}{2}\|u\|_{D^{\frac{1}{2}}}^{2}-\frac{\mu}{2}\|u\|_{L^{2}(\Omega)}^{2}-\|g\|_{L^{1}(\Omega)} \geq \frac{1}{2}\left(1-\frac{\mu}{\lambda_{1}}\right)\|u\|_{D^{\frac{1}{2}}}^{2}-C$.

It implies that

$$
\|u(t)\|_{D^{\frac{1}{2}}} \leq \frac{2 \lambda_{1}[\Phi(u(0))+C]}{\lambda_{1}-\mu}:=M, \quad \forall t \in\left[0, T_{\max }\right) .
$$

From (9) we conclude that $T_{\max }=+\infty$. Indeed, if $T_{\max }<+\infty$ then by (9) $\left\|u\left(T_{\max }-\frac{1}{n}\right)\right\|_{D^{\frac{1}{2}}} \leq M$ for $n \in \mathbb{Z}_{+}, n \geq \frac{1}{T_{\max }}$. By Proposition 1 there exists a constant $\mathrm{T}^{*}(\mathrm{M})$ (independent of $n$ ) such that the problem (1)-(3) has unique solution on $\left[T_{\max }-\frac{1}{n}, T_{\max }-\frac{1}{n}+T^{*}(M)\right)$, i. e. the solution $u(t)$ is defined on $\left[0, T_{\max }-\frac{1}{n}+T^{*}(M)\right)$. If $n>\frac{1}{T^{*}(M)}$ then $T_{\max }-\frac{1}{n}+T^{*}(M)>T_{\max }$, a contradiction to the definition of $T_{\max }$. in $D^{\frac{1}{2}}$.

Proposition 2 shows that the problem (1)-(3) generates a semigroup $S(t)$

Proposition 3. Assume that $f$ satisfies the conditions (4)-(7). Then the semigroup $S(t)$ has a compact connected global attractor in $D^{\frac{1}{2}}$.

Proof. Denote $E=\left\{w \in D^{\frac{1}{2}}:-P_{\alpha, \beta} w+f(x, y, z, w)=0\right\}$, the set of equilibrium points. If $w \in E$ then

$$
0=\|w\|_{D^{\frac{1}{2}}}^{2}+\int_{\Omega} f(x, y, z, w) w \mathrm{~d} x \mathrm{~d} y \mathrm{~d} z \geq\left(1-\frac{\mu}{\lambda_{1}}\right)\|w\|_{D^{\frac{1}{2}}}^{2}-\|g\|_{L^{1}(\Omega)},
$$

hence

$$
\|w\|_{D^{\frac{1}{2}}}^{2} \leq \frac{C \lambda_{1}}{\lambda_{1}-\mu}
$$

i. e. $E$ is bounded in $D^{\frac{1}{2}}$. It remains to prove that $S(t)$ is asymptotically compact in $D^{\frac{1}{2}}$. Assume that $u_{n}$ is a bounded sequence in $D^{\frac{1}{2}}$ and $t_{n} \rightarrow+\infty$. Fix $T>0$. Since $D^{\frac{1}{2}}$ is compactly imbedded in $L^{2}(\Omega)$ there exists a subsequence $n_{k}$ and an element $v \in D^{\frac{1}{2}}$ such that $v_{k}=S\left(t_{n_{k}}-T\right) u_{n_{k}} \rightarrow v$ in $D^{\frac{1}{2}}$ and $v_{k} \longrightarrow v$ in $L^{2}(\Omega)$ when $k \rightarrow+\infty$. We will prove that $S\left(t_{n_{k}}\right) u_{n_{k}}=S(T) v_{k}$ converges to $S(T) v$ strongly in $D^{\frac{1}{2}}$. Denoting $v_{k}(t)=S(t) v_{k}, v(t)=S(t) v$, or 
in other words

$$
\begin{gathered}
v_{k}(t)=\mathrm{e}^{-A t} v_{k}-\int_{0}^{t} \mathrm{e}^{-A(t-s)} f\left(x, y, z, v_{k}(s)\right) \mathrm{d} s \\
v(t)=\mathrm{e}^{-A t} v-\int_{0}^{t} \mathrm{e}^{-A(t-s)}(f(x, y, z, v(s))) \mathrm{d} s .
\end{gathered}
$$

We have

$$
\begin{aligned}
\left\|v_{k}(t)-v(t)\right\|_{D^{\frac{1}{2}}} & \left\|\mathrm{e}^{-A t}\left(v_{k}-v\right)-\int_{0}^{t} \mathrm{e}^{-A(t-s)}\left(f\left(x, y, z, v_{k}(s)\right)-f(x, y, z, v(s))\right) \mathrm{d} s\right\|_{D^{\frac{1}{2}}} \\
\leq & \left\|\mathrm{e}^{-A t}\left(v_{k}-v\right)\right\|_{D^{\frac{1}{2}}}+\int_{0}^{t}\left\|\mathrm{e}^{-A(t-s)}\left(f\left(x, y, z, v_{k}(s)\right)-f(x, y, z, v(s))\right)\right\|_{D^{\frac{1}{2}}} \mathrm{~d} s \\
\leq & C\left[t^{-\frac{1}{2}}\left\|v_{k}-v\right\|_{L^{2}(\Omega)}+\int_{0}^{t}(t-s)^{-\frac{1}{2}-\gamma_{0}} \|\left(f\left(x, y, z, v_{k}(s)\right)\right.\right. \\
& \left.\quad-f(x, y, z, v(s))) \|_{D^{-\gamma_{0}}} \mathrm{~d} s\right] \\
\leq & C\left[t^{-\frac{1}{2}}\left\|v_{k}-v\right\|_{L^{2}(\Omega)}+\int_{0}^{t}(t-s)^{-\frac{1}{2}-\gamma_{0}}\left\|v_{k}(s)-v(s)\right\|_{D^{\frac{1}{2}}} \mathrm{~d} s\right] .
\end{aligned}
$$

By a Gronwall's inequality we deduce that

$$
\left\|v_{k}(t)-v(t)\right\|_{D^{\frac{1}{2}}} \leq C t^{-\frac{1}{2}}\left\|v_{k}-v\right\|_{L^{2}(\Omega)} .
$$

In particular,

$$
\left\|v_{k}(T)-v(T)\right\|_{D^{\frac{1}{2}}} \leq C T^{-\frac{1}{2}}\left\|v_{k}-v\right\|_{L^{2}(\Omega)},
$$

which implies that $v_{k}(T) \rightarrow v(T)$ in $D^{\frac{1}{2}}$ when $k \rightarrow+\infty$ since $v_{k} \rightarrow v$ in $L^{2}(\Omega)$ when $k \rightarrow+\infty$.

Theorem 1. Assume that $f$ satisfies the conditions (4)-(7). Then the semigroup $S(t)$, defined by the problem (1)-(3), posseses a minimal compact connected global attractor in the space $S_{0}^{1}(\Omega)$. Moreover, for each $u_{0} \in S_{0}^{1}(\Omega)$ the corresponding solution $u(t)=S(t) u_{0}$ tends to the set $E$ of equilibrium points in $S_{0}^{1}(\Omega)$ as $t \rightarrow+\infty$.

Proof. The existence of the minimal compact connected global attractor $M$ results from the existence of the compact connected global attractor for the semigroup $S(t)$ established in Proposition 3. We will prove that $M \subset E$. Since $M=\cup_{u \in D^{\frac{1}{2}}} \omega(u)$, it suffices to show that if $v \in \omega(u)$ then $S(t) v=v$ for all $t>0$, i. e. $v \in E$. By definition of $\omega(u)$ there exists a sequence $t_{n}$ such that $\lim _{n \rightarrow \infty} t_{n}=+\infty$ and $S\left(t_{n}\right) u \rightarrow v$ in $D^{\frac{1}{2}}$ as $n$ tends to $+\infty$. In view of continuity and non-increasing property of $\Phi(S(t) u)$, we conclude that $\Phi(v)=\liminf _{t \rightarrow+\infty} \Phi(S(t) u)$. This means that $\Phi$ is a constant on $\omega(u)$. On the other hand, if $v \in \omega(u)$ then $S(t) v \in \omega(u)$. Therefore $\Phi(S(t) v)$ is independent of $t$. This in turn implies that $\left\|\frac{\mathrm{d}(S(t) v)}{\mathrm{d} t}\right\|_{D^{\frac{1}{2}}}=0$, or $S(t) v$ is independent of $t$. 


\section{Non-gradient systems}

In this section we consider the case when the nonlinear term satisfies other growth conditions, namely, there are constants $C$ and $p$ such that

$$
\begin{aligned}
& C\left(|u|^{p}-1\right) \leq f(x, y, z, u) u \leq C\left(|u|^{p}+1\right), \quad p>2, \\
& (u-v)(f(x, y, z, u)-f(x, y, z, v)) \geq-C(u-v)^{2} .
\end{aligned}
$$

Because, in general, $p$ may be a big number, the fixed point method for proving the existence of solutions in the second part is not valid here and we need to use the Galerkin method. The solution obtained here is only weak. Furthermore, the system in that case is no longer a gradient system. Under these assumptions the weak solutions of the problem (1)-(3) can be written as $u(t)=S(t) u_{0}$, where $S(t)$ is a continuous semigroup acting on $L^{2}(\Omega)$.

Definition 1. Let $T>0$ and $u_{0} \in L^{2}(\Omega)$. A function $u$ is called a weak solution of the problem $(1)-(3)$ on $(0, T)$ if

$$
\begin{aligned}
u \in & L^{p}\left(\Omega_{T}\right) \cap L^{2}\left((0, T) ; S_{0}^{1}(\Omega)\right) \cap C\left([0, T) ; L^{2}(\Omega)\right) ; \\
\frac{\partial u}{\partial t} \in & L^{2}\left((0, T) ; S^{-1}(\Omega)+L^{q}(\Omega)\right) ; u(0)=u_{0} ; \frac{1}{p}+\frac{1}{q}=1 ; \\
0= & \int_{0}^{T}\left\langle u_{t}, \varphi\right\rangle \mathrm{d} t+\int_{\Omega_{T}} f(x, y, z, u) \varphi \mathrm{d} x \mathrm{~d} y \mathrm{~d} z \mathrm{~d} t \\
& +\int_{\Omega_{T}}\left(\left(\nabla_{x} u, \nabla_{x} \varphi\right)+\left(\nabla_{y} u, \nabla_{y} \varphi\right)+|x|^{2 \alpha}|y|^{2 \beta}\left(\nabla_{z} u, \nabla_{z} \varphi\right)\right) \mathrm{d} x \mathrm{~d} y \mathrm{~d} z \mathrm{~d} t,
\end{aligned}
$$

where $\langle.,$.$\rangle denotes the pairing between S^{-1}(\Omega)$ and $S_{0}^{1}(\Omega)$, for all $\varphi \in$ $L^{p}\left(\Omega_{T}\right) \cap L^{2}\left((0, T) ; S_{0}^{1}(\Omega)\right)$.

Theorem 2. Assume that $f$ satisfies the conditions (10)-(11). Then the problem (1)-(3) has a unique weak solution satisfying

$$
\begin{aligned}
& \left.u \in C\left([0,+\infty) ; L^{2}(\Omega)\right)\right) \cap L^{2}\left((0,+\infty) ; S_{0}^{1}(\Omega)\right) \cap L_{\mathrm{loc}}^{p}\left((0,+\infty) ; L^{p}(\Omega)\right), \\
& \frac{\partial u}{\partial t} \in L_{\mathrm{loc}}^{2}\left((0,+\infty) ; S^{-1}(\Omega)\right)+L_{\mathrm{loc}}^{p^{\prime}}\left([0,+\infty) ; L^{p^{\prime}}(\Omega)\right) .
\end{aligned}
$$

Moreover, for each fixed the map $u_{0} \longrightarrow u(t)=S(t) u_{0}$ is continuous on $L^{2}(\Omega)$.

Proof. A) The existence. First, fix a positive finite number $T$. We find the approximate solutions $u_{n}(x, y, z, t)$ of the problem (1)-(3) in the form

$$
u_{n}(x, y, z, t)=\sum_{l=1}^{n} u_{n, l}(t) e_{l} .
$$

By substituting $u_{n}$ instead of $u$ into (1) then multiplying the obtained expression with $e_{j}$ and integrating over $\Omega$ we see that the functions $u_{n, j}(t)$ should satisfy the following problem

$$
\begin{aligned}
& \frac{\mathrm{d} u_{n, j}(t)}{\mathrm{d} t}+\lambda_{j} u_{n, j}(t)+\left(f\left(x, y, z, u_{n}(x, y, z, t)\right), e_{j}\right)_{L^{2}(\Omega)}=0, \\
& u_{n, j}(0)=\left(u_{0}, e_{j}\right)_{L^{2}(\Omega)}, \text { for } j=1, \ldots, n .
\end{aligned}
$$


By multiplying (12) with $u_{n, j}(t)$ then summing in $j$ from 1 to $n$ we have

$$
\frac{1}{2} \frac{\mathrm{d}\left\|u_{n}(t)\right\|_{L^{2}(\Omega)}^{2}}{\mathrm{~d} t}+\left\|u_{n}(t)\right\|_{S_{0}^{1}(\Omega)}^{2}+\int_{\Omega} f\left(x, y, z, u_{n}(t)\right) u_{n}(t) \mathrm{d} x \mathrm{~d} y \mathrm{~d} z=0 .
$$

From (10)-(11) we obtain

$$
\frac{1}{2} \frac{\mathrm{d}\left\|u_{n}(t)\right\|_{L^{2}(\Omega)}^{2}}{\mathrm{~d} t}+\left\|u_{n}(t)\right\|_{S_{0}^{1}(\Omega)}^{2}+\left\|u_{n}(t)\right\|_{L^{p}(\Omega)}^{p} \leq \mu\left\|u_{n}(t)\right\|_{L^{2}(\Omega)}^{2}+C .
$$

By a Gronwall inequality

$$
\begin{aligned}
\left\|u_{n}(t)^{2}\right\|_{L^{2}(\Omega)} & \leq \mathrm{e}^{-\left(\lambda_{1}-\mu\right) t}\left\|u_{n}(0)\right\|_{L^{2}(\Omega)}^{2}+\frac{C\left(1-\mathrm{e}^{-\left(\lambda_{1}-\mu\right) t}\right)}{\lambda_{1}-\mu} \\
& \leq \mathrm{e}^{-\left(\lambda_{1}-\mu\right) t}\left\|u_{0}\right\|_{L^{2}(\Omega)}^{2}+\frac{C\left(1-\mathrm{e}^{-\left(\lambda_{1}-\mu\right) t}\right)}{\lambda_{1}-\mu}
\end{aligned}
$$

and therefore by (14)

$$
\begin{aligned}
& \frac{1}{2} \frac{\mathrm{d}\left\|u_{n}(t)\right\|_{L^{2}(\Omega)}^{2}}{\mathrm{~d} t}+\left\|u_{n}(t)\right\|_{S_{0}^{1}(\Omega)}^{2}+C\left\|u_{n}(t)\right\|_{L^{p}(\Omega)}^{p} \\
& \quad \leq \mathrm{e}^{-\left(\lambda_{1}-\mu\right) t}\left\|u_{0}\right\|_{L^{2}(\Omega)}^{2}+\frac{C\left(1-\mathrm{e}^{-\left(\lambda_{1}-\mu\right) t}\right)}{\lambda_{1}-\mu}, \forall t \in[0, T] .
\end{aligned}
$$

Integrating in $t$ from 0 to $T$ gives

$$
\left\|u_{n}(T)\right\|_{L^{2}(\Omega)}^{2}+2 \int_{0}^{T}\left\|u_{n}(t)\right\|_{S_{0}^{1}(\Omega)}^{2} \mathrm{~d} t+C\left\|u_{n}\right\|_{L^{p}(\Omega)}^{p} \leq C\left(1+\left\|u_{0}\right\|_{L^{2}(\Omega)}^{2}\right) .
$$

This means that $u_{n}(t)$ is a bounded sequence in $\left.L^{\infty}\left((0, T) ; L^{2}(\Omega)\right)\right)$, $L^{2}\left((0, T) ; S_{0}^{1}(\Omega)\right)$ and $L^{p}\left((0, T) ; L^{p}\left(\Omega_{T}\right)\right)$. From the hypotheses $(10)-(11)$ we conclude that $f\left(x, y, z, u_{n}\right)$ is a bounded sequence in $L^{q}\left(\Omega_{T}\right)$. Next, from the equation

$$
\frac{\partial u_{n}}{\partial t}=P_{\alpha, \beta} u_{n}-f\left(x, y, z, u_{n}\right)
$$

it follows that the sequence $\frac{\partial u_{n}}{\partial t}$ is bounded in $L^{2}\left((0, T) ; S^{-1}(\Omega)\right)+L^{q}\left(\Omega_{T}\right)$. From all above said we can choose functions $u, v$ satisfying

$u \in L^{2}\left((0, T) ; S_{0}^{1}(\Omega)\right) \cap L^{p}\left(\Omega_{T}\right) ; \quad \frac{\partial u}{\partial t} \in L^{2}\left((0, T) ; S^{-1}(\Omega)\right)+L^{q}\left(\Omega_{T}\right) ; \quad v \in L^{q}\left(\Omega_{T}\right)$ and a subsequence $n_{k}$ such that

$$
\begin{aligned}
u_{n_{k}} & \rightarrow u \quad \text { in } L^{2}\left((0, T) ; S_{0}^{1}(\Omega)\right), \\
u_{n_{k}} & \rightarrow u \quad \text { in } L^{p}\left(\Omega_{T}\right), \\
f\left(x, y, z, u_{n_{k}}\right) & \rightarrow v \quad \text { in } L^{q}\left(\Omega_{T}\right), \\
\frac{\partial u_{n_{k}}}{\partial t} & \rightarrow \frac{\partial u}{\partial t} \quad \text { in } L^{2}\left((0, T) ; S^{-1}(\Omega)\right),
\end{aligned}
$$

as $n_{k} \rightarrow+\infty$. By Theorem 3.1 in [13] (page 33) we see that $u \in$ $C\left([0, T] ; L^{2}(\Omega)\right)$.

Next, we show that $f(x, y, z, u)=v$. Indeed, by Theorem 2.1 of [14] (page 271) we can assume (passing again to a subsequence if necessary) that 
$f\left(x, y, z, u_{n_{k}}\right)$ converges almost everywhere to $f(x, y, z, u)$ as $n_{k} \rightarrow+\infty$. Combining with the fact that $f\left(x, y, z, u_{n_{k}}\right)$ is bounded in $L^{q}\left(\Omega_{T}\right)$ we conclude that $f\left(x, y, z, u_{n_{k}}\right) \rightarrow f(x, y, z, u)$ in $L^{q}\left(\Omega_{T}\right)$ as $n_{k} \rightarrow+\infty$. Hence $f(x, y, z, u)=v$.

The proof of the fact that $u(0)=u_{0}$ goes without difficulty as in the initial boundary value problem for semilinear parabolic equations.

Since $T$ does not depend on $u_{0}$ it is clear that $u(t)$ can be extended as solution defined globally on $[0, \infty)$.

B) The uniqueness and continuous dependence. Assume that $u, v$ are two solutions of the problem (1)-(3) with initial values $u_{0}, v_{0}$. Put $w=u-v$. Then $w$ solve the following problem

$$
\begin{gathered}
\frac{\partial w}{\partial t}-P_{\alpha, \beta} w+f(x, y, z, u)-f(x, y, z, v)=0,(x, y, z) \in \Omega, t>0, \\
w(x, y, z, t)=0,(x, y, z) \in \partial \Omega, t>0, \\
w(x, y, z, 0)=u_{0}(x, y, z)-v_{0}(x, y, z),(x, y, z) \in \Omega .
\end{gathered}
$$

Multiplying both sides of (18) by $2 w$ and then integrating by parts we obtain

$$
\begin{aligned}
0 & =\frac{\mathrm{d}\|w\|_{L^{2}(\Omega)}^{2}}{\mathrm{~d} t}+2\|w\|_{S_{0}^{1}(\Omega)}^{2}+2 \int_{\Omega}(u-v)(f(x, y, z, u)-f(x, y, z, v)) \mathrm{d} x \mathrm{~d} y \mathrm{~d} z \\
& \geq \frac{\mathrm{d}\|w\|_{L^{2}(\Omega)}^{2}}{\mathrm{~d} t}+2\|w\|_{S_{0}^{1}(\Omega)}^{2}-2 C\|w\|_{L^{2}(\Omega)}^{2} .
\end{aligned}
$$

Therefore by applying a Gronwall inequality we deduce that

$$
\|w(t)\|_{L^{2}(\Omega)} \leq\|w(0)\|_{L^{2}(\Omega)} \mathrm{e}^{2 C t} .
$$

Now the uniqueness and continuous dependence follow from the last inequality.

Theorem 2 shows that the problem (1)-(3) generates a continuous semigroup $S(t)$ in $L^{2}(\Omega)$.

Theorem 3. Assume that $f$ satisfies the conditions (10)-(11). Then the problem (1)-(3) defines a continuous semigroup $S(t): L^{2}(\Omega) \longrightarrow L^{2}(\Omega)$, which posseses a compact connected global attractor in the space $L^{2}(\Omega)$.

Proof. By (17) there exists a constant $R$ with the property that for any $u_{0} \in$ $L^{2}(\Omega)$ we can find a number $t_{0}\left(\left\|u_{0}\right\|_{L^{2}(\Omega)}\right)$ such that $\left\|u_{n}(t)\right\|_{L^{2}(\Omega)} \leq R$ for all $n$ and $t \geq t_{0}$. Therefore integrating (16) in $t$ over $(s, s+1)$ we obtained

$$
\int_{s}^{s+1}\left(\left\|u_{n}(t)\right\|_{S_{0}^{1}(\Omega)}^{2}+C\left\|u_{n}(t)\right\|_{L^{p}(\Omega)}^{p}\right) \mathrm{d} t \leq C+\frac{1}{2} R^{2}, \quad \forall n \text { and } s \geq t_{0} .
$$

By noting that $C\left(|u|^{p}-1\right) \leq F(x, y, z, u) \leq C\left(|u|^{p}+1\right)$ we have

$$
\int_{s}^{s+1}\left[\left\|u_{n}(t)\right\|_{S_{0}^{1}(\Omega)}^{2}+\int_{\Omega} F\left(x, y, z, u_{n}(t)\right) \mathrm{d} x \mathrm{~d} y \mathrm{~d} z\right] \mathrm{d} t \leq C, \forall n \text { and } s \geq t_{0} .
$$

On the other hand, since $u_{n}(t) \in S_{0}^{1}(\Omega) \cap L^{p}(\Omega)$ we have

$$
\frac{\mathrm{d}}{\mathrm{d} t}\left[\left\|u_{n}(t)\right\|_{S_{0}^{1}(\Omega)}^{2}+\int_{\Omega} F\left(x, y, z, u_{n}(t)\right) \mathrm{d} x \mathrm{~d} y \mathrm{~d} z\right]=-\left\|\frac{\partial u_{n}(t)}{\partial t}\right\|_{L^{2}(\Omega)}^{2} \leq 0 .
$$


From (19), (20) it follows that

$$
\left\|u_{n}(t)\right\|_{S_{0}^{1}(\Omega)}^{2}+\int_{\Omega} F\left(x, y, z, u_{n}(t)\right) \mathrm{d} x \mathrm{~d} y \mathrm{~d} z \leq C, \quad \forall n \text { and } s \geq t_{0}+1,
$$

from which we deduce that

$$
\left\|u_{n}(t)\right\|_{S_{0}^{1}(\Omega)}^{2}+\left\|u_{n}(t)\right\|_{L^{p}(\Omega)}^{p} \leq C, \quad \forall n \text { and } t \geq t_{0}+1 .
$$

Passing to the limit when $n \rightarrow+\infty$ we get

$$
\|u(t)\|_{S_{0}^{1}(\Omega)}+\|u(t)\|_{L^{p}(\Omega)} \leq C, \quad \forall t \geq t_{0}+1 .
$$

The final estimate shows that the set $B_{C}(0)=\left\{u \in S_{0}^{1}(\Omega) \cap L^{p}(\Omega):\|u\|_{S_{0}^{1}(\Omega)}+\right.$ $\left.\|u\|_{L^{p}(\Omega)} \leq C\right\}$ is an absorbing set for the semigroup $S(t)$. Now the existence of the connected compact global attractor $A$ is resulted from the compact imbedding of $S_{0}^{1}(\Omega)$ into $L^{2}(\Omega)$, (in fact $A=\omega\left(B_{C}(0)\right)$ ).

Remark 2. If $f \in C^{1}(\Omega \times \mathbb{R} ; \mathbb{R})$ and $\frac{\partial f(x, y, z, u)}{\partial u} \geq-C$ then $f$ satisfies the condition (11).

\section{Acknowledgments}

The authors express thanks to NAFOSTED for the financial support.

\section{References}

[1] Chueshov, I.D.: Introduction to the Theory of Infinite-Dimensional Dissipative Systems. ACTA Scientific Publishing House, Kharkov (2002)

[2] Hale, J.K.: Asymptotic behavior of dissipative systems. In: Mathematical Surveys and Monographs, vol. 25. American Mathematical Society, Providence (1988)

[3] Raugel, G.: Global attractors in partial differential equations. In: Handbook of Dynamical Systems, vol. 2, pp. 885-892. North-Holland, Amsterdam (2002)

[4] Anh, C.T., Hung, P.Q., Ke, T.D., Phong, T.T.: Global attractor for a semilinear parabolic equation involving the Grushin operator. Electron. J. Differ. Equ. 2008(32), 1-11 (2008)

[5] Anh, C.T., Ke, T.D.: Existence and continuity of global attractors for a degenerate semilinear parabolic equation. Electron. J. Differ. Equ. 2009(61), 1-13 (2009)

[6] Thuy, P.T., Tri, N.M.: The phenomenon of critical exponents of boundary value problem for semilinear elliptic degenerate differential equations. In: Proceedings of the International Conference on Partial Differential Equations, Moscow (2009)

[7] Thuy, P.T., Tri, N.M.: Nontrivial solutions to boundary value problems for semilinear strongly degenerate elliptic differential equations. Nonlinear Differ. Equ. Appl. 19(3), 279-298 (2012) 
[8] Franchi, B., Lanconelli, E.: An embedding theorem for Sobolev spaces related to nonsmooth vector fields and Harnack inequality. Comm. Partial Differ. Equ. 9, 1237-1264 (1984)

[9] Tri, N.M.: Recent results in the theory of semilinear elliptic degenerate differential equations. Vietnam J. Math. 37, 387-397 (2009)

[10] Tri, N.M.: Semilinear degenerate elliptic differential equations, local and global theories. Lambert Academic Publishing, Saarbrücken (2010)

[11] Bergh, J., Lofstrom, J.: Interpolation spaces. Springer, Berlin (1976)

[12] Henry, D.: Geometric theory of semilinear parabolic equations. In: Lecture Notes Math., vol. 840 (1981)

[13] Lions, J.L., Magenes, B.: Non-homogeneous limit problems and applications (in Russian), vol. 1. Mir, Moscow (1971)

[14] Temam, R.: Navier-Stokes equations. Theory and numerical analysis. In: Studies in Mathematics and its Applications. North-Holland Publishing Co., Amsterdam (1977)

P. T. Thuy and N. M. Tri

Institute of Mathematics

18 Hoang Quoc Viet

Cau Giay

10307 Hanoi

Vietnam

e-mail: triminh@math.ac.vn

Received: 24 May 2012.

Accepted: 21 October 2012. 